\title{
A Hybrid Scheme for Object Allocation in a Distributed Object-Storage System*
}

\author{
Fang Wang ${ }^{* *}$, Shunda Zhang, Dan Feng, Hong Jiang, Lingfang Zeng, \\ and Song Lv \\ Key Laboratory of Data Storage System, Ministry of Education, \\ School of Computer, Huazhong University of Science and Technology, Wuhan, China \\ wangfang@mail.hust.edu.cn, zhangshunda@163.com
}

\begin{abstract}
The object-based storage system, in which files are mapped onto one or more data objects stored on Object-Based Storage Devices (OSDs), has distributed storage system architecture. In such a system, the policy for object allocation is a critical aspect affecting the overall systems performance. Hashing and fragment-strip are two common techniques used for managing objects, but both have their disadvantages, and advantages, e.g. hashing achieves good workload balance and provide rather high effectiveness in allocating data, but it can not provide readily available parallelism for large file; fragment-strip takes full advantage device parallelism, simplifies the clients' operations, but this policy is not fit for small file. In this paper, we present an efficient algorithm that combines the advantages of these two approaches while avoiding their shortcomings. The key factors which can impact the performance of the whole system in the objects allocation are also be discussed.
\end{abstract}

\section{Introduction}

Object-based storage systems represent files as sets of objects stored on self-managed Object-Based Storage Devices (OSDs). By distributing the objects across many devices, these systems have the potential to provide high throughput, reliability, availability and scalability [1]. Much research has gone into hierarchy management, scalability, and availability of distributed file systems in projects such as AFS [3], Lustre [8], GFS [11], Coda [12] and GPFS [13], but relatively little research has been aimed at improving the efficiency of objects allocation in large scale object-based storage systems. The algorithm used for object allocation determines the performance of the system at the beginning of the communication process. It affects the workload among the devices, and it also influences the OSD-level parallelism of the objectbased storage system.

The object-based storage model is emerging as architecture for distributed storage systems. Traditionally, metadata and data are managed by the same file system, on the same machine, and stored on the same device [3]. For efficiency,

\footnotetext{
* This work was supported by the National Basic Research Program of China (973 Program) under Grant No. 2004CB318201, the National Science Foundation of China under Grant No.60303032.

** Corresponding author.
} 
metadata is often stored physically close to the data it describes [4]. In some modern distributed file systems, data is stored on devices that can be directly accessed through the network, while metadata is managed separately by one or more specialized metadata servers [5].

Currently, most approaches to object allocation employ one of two techniques. The first one, which we call hashing [2], allocates a file to one device by using hashing functions that map file IDs to OSD IDs. This approach converts a file to one object and sends it to only one device. The second object allocation technique, which we call fragment-strip or fragment-mapping [2], uses equal-sized fragments of each file to widely distribute the file among the OSDs.

Our object allocation scheme combines the best aspects of hashing and fragmentstrip. In the algorithm, when the file is small, it is converted to a single object and directly mapped to an OSD by hashing. If the file is large, it is converted to multiple objects and each object will be distributed to an OSD.

\section{Related Works}

To improve the scalability of hashing, a self-adaptive hashing scheme is presented in [6]. To reduce the cost of adaptation and continue to exploit the high effectiveness of hash functions, the self-adapt hashing policy is designed to improve scalability.

In OBFS (a file system for object-based storage devices) [1], the boundary of small objects and large objects is set at $512 \mathrm{~KB}$. The workload characteristics of a highperformance distributed file system from Lawrence Livermore National Laboratory (LLNL) [7] were analyzed as an example of large-scale distributed file systems [1]. OBFS provides most of the files are larger than $4 \mathrm{~KB}$ and the majority of all files are distributed between $32 \mathrm{~KB}$ and $8 \mathrm{MB}$. Those files that are smaller than $4 \mathrm{~KB}$ (a typical block size for a general-purpose file system) only account for a very small portion of the total files.

In the Panasas storage cluster [9], if a file is smaller than $64 \mathrm{~KB}$, it will be mirrored on the first two component objects (RAID 1). If the file is larger than $64 \mathrm{~KB}$, it will use additional component objects, up to full stripe worth (RAID 5). That means that $64 \mathrm{~KB}$ is the boundary distinguishing small files from large files, and that objects are not larger than $64 \mathrm{~KB}$. The Lustre cluster file system [8] logical object volume management (LOVM) manages the objects as RAID.

\section{Algorithm Design}

\subsection{The Boundary of Small and Large File}

According to OBFS [1], this boundary of small and large files should be 512KB. The OBFS's conclusion was based on the analysis of LLNL [7] workload characteristics. In the LLNL [7] workload, we estimate that about $85 \%$ of all objects will be of size $512 \mathrm{~KB}$ and $15 \%$ of all objects will be of size smaller than $512 \mathrm{~KB}$. We will refer to files that are smaller than $512 \mathrm{~KB}$ as small objects and the rest as large objects. Small objects and large objects are treated differently by the object allocation algorithm. 


\subsection{The Optimal Number of Objects Mapped from One File}

The relationship between the number of OSDs and parallelism is quite complex. More devices provide more transfer channels and data can be transferred in parallel. However, increasing connections can also bring down the performance, because establishing connections takes time, especially when connections are numerous. Thus, while increasing the number of devices mapping to the same file improves parallelism, it consumes extra resources of the system.

We can describe the relationship described above with the following formula:

$$
\frac{T_{p}}{T}=\frac{n \times a+\frac{1}{n} \times b+\delta(t)}{a+b}
$$

$T p$ : The time of transferring file with multiple objects in parallel.

$T$ : The time of transferring file sequentially.

$n$ : Number of objects mapped to a large file.

$a$ : The sum of sender overhead, receiver overhead and the time of flight of transferring a file.

$b$ : The time for transferring a whole file to a single device.

$\delta(t)$ : Other delays of objects transmission, it is an amendment factor of the formula.

Numerator of the formula is made up of three terms, which are $n^{*} a, b / n$ and $\delta(t)$. The term $n * a$ means connecting to $\mathrm{n}$ devices costs $\mathrm{n}$ times of the overhead connecting to a single device. And the $b / n$ indicates that $\mathrm{n}$ devices' parallel working can make bandwidth $\mathrm{n}$ times wider than the single-devices situation.

Let's review the performance parameters of interconnection networks.

Depending on whether it is an SAN, LAN or WAN, the relative lengths of the time of flight and transmission may be quite different from those shown here, based on a presentation by Greg Papadopoulos of Sun Microsystems. [10]

Total latency $=$ Sender overhead + Time of flight $+($ Message size $/$ Bandwidth $)+$ Receiver overhead

Notice that the time of flight for SANs is so short relative to overhead that it can be ignored, yet in WANs, time of flight is so long that sender and receiver overheads can be ignored. Thus, we can simplify the performance equation by combining sender overhead, receiver overhead, and time of flight into a single term called Overhead:

$$
\text { Total latency } \approx \text { Overhead }+(\text { Message size / Bandwidth })
$$

In our formula:

$$
a=\text { Overhead, } b=(\text { Message size } / \text { Bandwidth })
$$

Although the $\delta(t)$ has some relationship with $n$, their relationship is rather loose. That is to say, we can simplify $\delta(t)$ to a variable c that is irrelevant to $n$.

We can simplify our formula by replacing $\delta(t)$ with c: 


$$
\frac{T_{p}}{T}=n \times \frac{a}{a+b}+\frac{1}{n} \times \frac{b}{a+b}+\frac{c}{a+b}
$$

The reciprocal of the above formula, $T / T_{p}$ is the speedup in file transfer time as a result of parallelism. To maximize the speedup is equivalent to minimizing the above formula for $T_{p} / T$. Since we are interested in how to best map a file into objects, namely, determining an optimal $\mathrm{n}$, for data transfer, although $\mathrm{a}, \mathrm{b}$ are not constants, they are irrelevant to $\mathrm{n}$. We now show that the sum of the two terms containing $\mathrm{n}$ has a minimum when $n=\sqrt{b / a}$, as follows.

Let $F(n)={ }_{n \times \frac{a}{a+b}}+\frac{1}{n} \times \frac{b}{a+b}$, and solve for $\frac{d F(n)}{d n}=0$, we have $n=\sqrt{b / a}$. Since $\frac{d^{2} F(n)}{d n^{2}}>0, F(n)$ has a global minimum at $n=\sqrt{b / a}$. Therefore,

$$
\frac{T_{p}}{T} \geq \frac{2 \sqrt{a b}}{a+b}+\frac{c}{a+b}
$$

The Overhead is the time for the processor to inject the message into the network, including both hardware and software components. For pedagogic reasons, we assume that Overhead is not dependent on message size. (Typically, only very large messages have larger overhead.) So we can assume that a network with a bandwidth of $1000 \mathrm{Mbits} / \mathrm{second}$ has an Overhead of 80 microseconds [10]. This situation is very common in today's network, and we use this representative case to estimate the parameter we need. As the factor $\mathrm{c} /(\mathrm{a}+\mathrm{b})$ do not impact $\mathrm{n}$, we can ignore it.

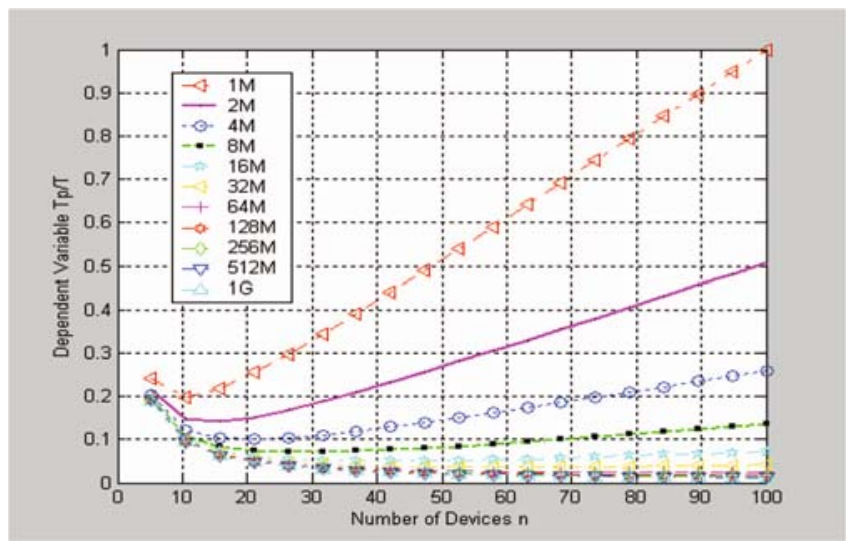

Fig. 1. The Relationship Curve of $T_{p} / T$ and $\mathrm{n}$

Figure 1 describes the relationship $T_{p} / T$ and Number of Devices $\mathrm{n}$ according to formula (6). Figure 1 shows that for $1 \mathrm{MB}$ and $2 \mathrm{MB}$ files, when $\mathrm{n}>=10$ the change in $T_{p} / T$ is not distinguishable. And for $4 \mathrm{MB}, 8 \mathrm{MB}$ and $16 \mathrm{MB}$, when $\mathrm{n}>=20$ the change in $T_{p} / T$ is not distinguishable. So do files above $32 \mathrm{MB}$ when $\mathrm{n}>=40$. We can arrive at a conclusion: $n=10$ (1MB-2MB), $n=20$ (4MB-16MB), $n=40$ (32MB-1GB). 


\subsection{How to Select OSDs for Parallel Transmission}

An object-based storage system typically has hundreds of OSDs. How to select OSDs for parallel transmission of large files from numerous devices? Random choice is a good idea, which is easy to implement and can always keep workload balanced. However, it can not ensure that the fastest devices are fully utilized. We can sort the devices by some parameters such as speed, free-capacity and so on, then select the first $\mathrm{n}(10,20$ or 40) devices. This algorithm makes sure that the best-conditioned devices are used first. However, sorting hundreds of OSDs is a time consuming task for the system. Does it affect the performance to some degree? We will carry out an asymptotic time complexity analysis of the sorting algorithm to address such questions.

In a bubble sort algorithm, the time complexity of searching the first $\mathrm{n}$ items from a total of $\mathrm{N}$ items is:

$$
\sum_{i=1}^{n}(N-i)=\frac{2 N-(n+1)}{2} \times n
$$

The time complexity: $T(n)=O\left(n^{2}\right)$ and $T(N)=O(N)$. So $n$ impact the time complexity more noticeably than $N$. Because $n$ is small $(10,20$ or 40$)$ and MDS always has high-capacity memory and high-performance CPUs, sorting algorithm will not affect the performance much. We can use $\bar{n}=(10+20+40) / 3 \approx 20$ instead of $n$ to simplify our module.

\subsection{Objects Allocation Algorithm Details}

The object allocation algorithm proposed here is based on sorting those devices by some parameters, such as OSD types, busy status, free capacity, partitions in the device and IP address. A pseudo-code of the algorithm is presented as Figure 2. The basic idea behind the algorithm is to find those best OSDs according to the size of files.

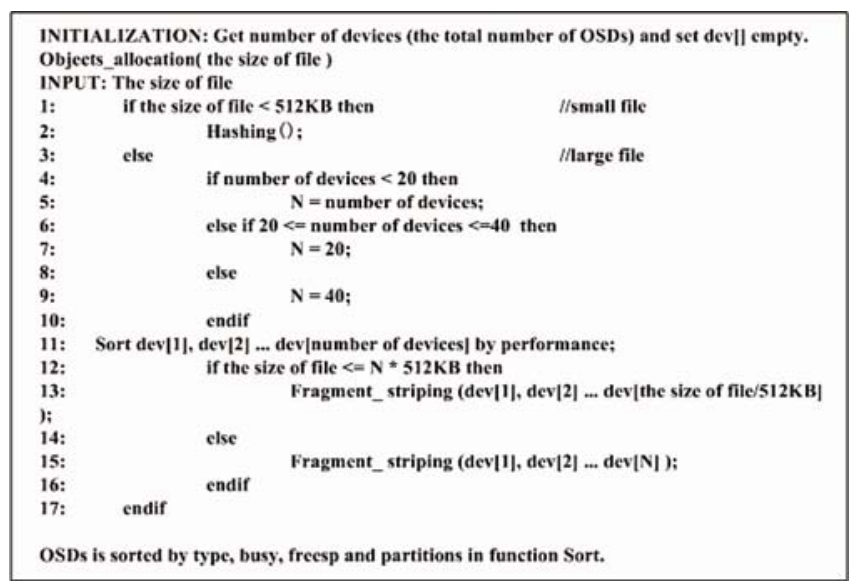

Fig. 2. Objects Allocation Algorithm 


\section{Simulation Results}

\subsection{Experimental Setup}

All of the experiments were executed on a PC with a $2.4 \mathrm{GHZ}$ Intel Celeron CPU and $512 \mathrm{MB}$ of RAM, running Red Hat Linux, kernel version 2.4.20. We used Matlab as the simulator. Matlab first generated an array of random numbers chosen from the exponential distribution of the file sizes. Then our algorithm (Section 3.4) was applied to estimate the response time of the system. We implemented the algorithm in Matlab's M file. The parameter a (Overhead) was assumed to be $80 \mu$ s and network bandwidth was assumed to be $1000 \mathrm{Mb} / \mathrm{s}$ (Section 3.2). Devices after being sorted should reduce the total response time (Section 3.3). According to the number of OSDs, the simulator considered the following situations: 16 OSDs, 32 OSDs, and 64 OSDs.

\subsection{Results}

Figures 3 and 4 show the simulation results with 16 OSDs, 32 OSDs and 64 OSDs. In each virtual system, we measure response times and compare among results from the hashing scheme, the fragment-strip policy and our algorithm.

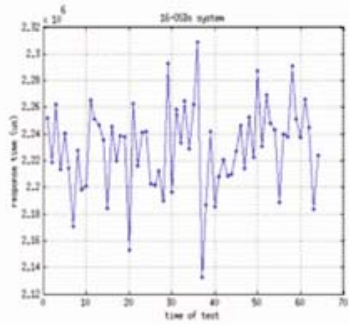

(a)

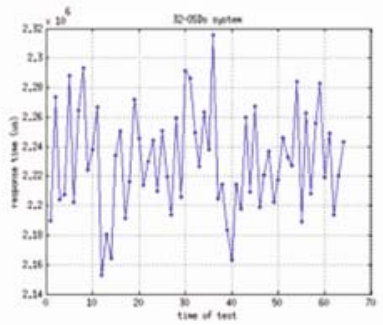

(b)

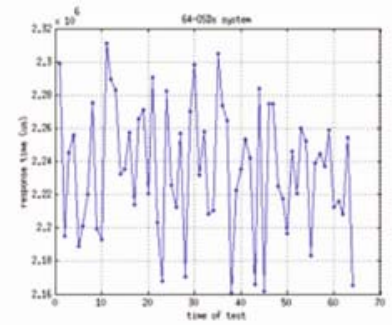

(c)

Fig. 3. Response time of the 16-OSDs (a), 32-OSDs (b) and 64 OSDs (c) system

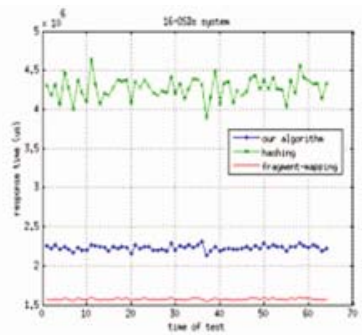

(a)

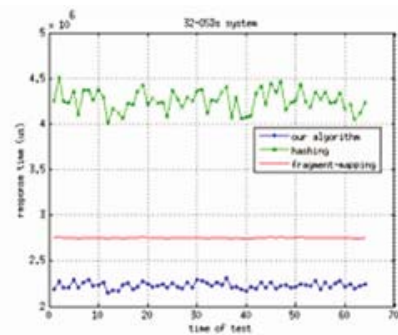

(b)

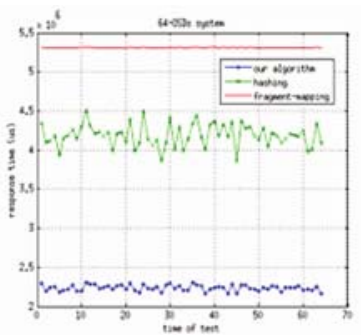

(c)

Fig. 4. Comparing our algorithm, hashing and fragment-strip in the 16-OSDs (a), 32-OSDs (b) and 64-OSDs (c) system 


\subsection{Analysis and Discussion of Experimental Results}

During evaluation process, we confirmed that the file sizes generated by Matlab conforms the description of file sizes from LLNL [7] (Section 2) fairly.

Tables 1, 2 and 3 show the mean value, standard deviation, and max and min value of the three algorithms' response time in different object-based storage systems. In the 16-OSDs system, the mean value of fragment-strip's response time is the smallest of the three algorithms. However, when the number of devices increases, the fragment-strip consumes more and more response time because of the increasing the Overhead. In the 32-OSDs system, the fragment-strip's mean value of response time is larger than our algorithm's. In the 64-OSDs system, the fragmentstrip's mean value of response time becomes larger than the other two. The response time of hashing is between 4 and 4.6 seconds, our algorithm's response time is between 2 and 2.3 seconds. They have not been changed much in the three different cases.

Our algorithm is faster than hashing because it makes good use of parallelism. The standard deviation of fragment-strip's response time decreases while the number of devices increases. And the standard deviation of hashing and fragment-strip is relatively steady. Our algorithm's standard deviation is smaller than hashing and larger than fragment-strip. As a whole, our algorithm is steadier than the others. And it performs best when the object-based storage system has many OSDs.

Table 1. The statistical data of our algorithm

\begin{tabular}{|c|c|c|c|c|}
\hline OSDs & $\begin{array}{c}\text { Mean value } \\
(\mu \mathrm{s})\end{array}$ & $\begin{array}{c}\text { Standard deviation } \\
(\mu \mathrm{s})\end{array}$ & $\begin{array}{c}\text { Max value } \\
(\mu \mathrm{s})\end{array}$ & $\begin{array}{c}\text { Min value } \\
(\mu \mathrm{s})\end{array}$ \\
\hline 16 & $2.22947 \mathrm{e}+06$ & $3.30344 \mathrm{e}+04$ & $2.30907 \mathrm{e}+06$ & $2.13224 \mathrm{e}+06$ \\
\hline 32 & $2.23067 \mathrm{e}+06$ & $3.51447 \mathrm{e}+04$ & $2.31596 \mathrm{e}+06$ & $2.15275 \mathrm{e}+06$ \\
\hline 64 & $2.23583 \mathrm{e}+06$ & $3.87005 \mathrm{e}+04$ & $2.31128 \mathrm{e}+06$ & $2.16086 \mathrm{e}+06$ \\
\hline
\end{tabular}

Table 2. The statistical data of hashing

\begin{tabular}{|c|c|c|c|c|}
\hline OSDs & $\begin{array}{c}\text { Mean value } \\
(\mu \mathrm{s})\end{array}$ & $\begin{array}{c}\text { Standard deviation } \\
(\mu \mathrm{s})\end{array}$ & $\begin{array}{c}\text { Max value } \\
(\mu \mathrm{s})\end{array}$ & $\begin{array}{c}\text { Min value } \\
(\mu \mathrm{s})\end{array}$ \\
\hline 16 & $4.27464 \mathrm{e}+06$ & $1.38243 \mathrm{e}+05$ & $4.64453 \mathrm{e}+06$ & $3.88316 \mathrm{e}+06$ \\
\hline 32 & $4.25589 \mathrm{e}+06$ & $1.18006 \mathrm{e}+05$ & $4.51130 \mathrm{e}+06$ & $4.01033 \mathrm{e}+06$ \\
\hline 64 & $4.18744 \mathrm{e}+06$ & $1.44812 \mathrm{e}+05$ & $4.51206 \mathrm{e}+06$ & $3.85776 \mathrm{e}+06$ \\
\hline
\end{tabular}

Table 3. The statistical data of fragment-strip

\begin{tabular}{|c|c|c|c|c|}
\hline OSDs & $\begin{array}{c}\text { Mean value } \\
(\mu \mathrm{s})\end{array}$ & $\begin{array}{c}\text { Standard deviation } \\
(\mu \mathrm{s})\end{array}$ & $\begin{array}{c}\text { Max value } \\
(\mu \mathrm{s})\end{array}$ & $\begin{array}{c}\text { Min value } \\
(\mu \mathrm{s})\end{array}$ \\
\hline 16 & $1.57276 \mathrm{e}+06$ & $8.64023 \mathrm{e}+03$ & $1.59588 \mathrm{e}+06$ & $1.54829 \mathrm{e}+06$ \\
\hline 32 & $2.75187 \mathrm{e}+06$ & $3.68768 \mathrm{e}+03$ & $2.75985 \mathrm{e}+06$ & $2.74420 \mathrm{e}+06$ \\
\hline 64 & $5.30830 \mathrm{e}+06$ & $2.26269 \mathrm{e}+03$ & $5.31338 \mathrm{e}+06$ & $5.30315 \mathrm{e}+06$ \\
\hline
\end{tabular}




\section{Conclusion and Future Work}

In this paper, we present a hybrid algorithm of hashing and fragment-strip, which combines the best aspects of these two algorithms while avoiding their disadvantages. Fragment-strip's good scalability is retained reasonably well, while the high efficiency of hashing makes its presence felt in our algorithm. We combine the two popular objects-allocation approaches at $512 \mathrm{~KB}$, the boundary of small and large file. We calculate two key factors in our algorithm, namely, the optimal number of objects mapped from one file and the scope of selecting OSDs for parallel transmission. Simulation results validate the correctness of the parameters we have calculated. Our object-allocation algorithm consumes lest time when there are numerous OSDs and its performance does not change much when the total number of devices increases.

As future work, we plan to finish the Object-Based Storage System. We will also test the algorithm in the real system instead of the simulation environment created by Matlab.

\section{References}

1. Feng Wang, Scott A. Brandt, and Ethan L. Miller, Darrell D. E. Long.: OBFS: A File System for Object-based Storage Devices. In 21st IEEE / 12th NASA Goddard Conference on Mass Storage Systems and Technologies (MSST2004), College Park, MD, April 2004.

2. Lan Xue, Yong Liu.: MDS Functionality Analysis. December 4, 2001.

3. J. H. Morris, M. Satyanarayanan, M. H. Conner, J. H. Howard, D. S. H. Rosenthal, and F. D. Smith.: Andrew: A distributed personal computing environment. Communications of the ACM, 29(3):184-201, Mar. 1986.

4. M. K. McKusick,W. N. Joy, S. J. Leffler, and R. S. Fabry.: A fast file system for UNIX. ACM Transactions on Computer Systems, 2(3):181-197, Aug. 1984.

5. G. A. Gibson and R. V. Meter.: Network attached storage architecture. Communications of the ACM, 43(11):37-45, 2000.

6. M. Spasojevic and M. Satyanarayanan.: An Empirical Study of a Wide-Area Distributed File System. ACM Transactions on Computer Systems 14(2) May 1996.

7. D. Roselli, J. Lorch, and T. Anderson.: A comparison of file system workloads. In Proceedings of the 2000 USENIX Annual Technical Conference, June 2000.

8. Peter J. Braam (with others): The Lustre Storage Architecture. Cluster File Systems, Inc. http://www.clusterfs.com August 2003.

9. J.R. Moase.: Panasas Storage Cluster \& Object Storage Overview. www.panasas.com October 2004

10. John L. Hennessy, David A. Patterson.: Computer Architecture A Quantitative Approach (Third Edition).

11. S. R. Soltis, T. M. Ruwart, and M. T. O'Keefe.: The Global File System. In Proceedings of the 5th NASA Goddard Conference on Mass Storage Systems and Technologies, College Park, MD, 1996.

12. M. Satyanarayanan, J. J. Kistler, P. Kumar, M. E. Okasaki, E. H. Siegel, and D. C. Steere.: Coda: A highly available file system for a distributed workstation environment. IEEE Transactions on Computers, 39(4):447-459, 1990.

13. F. Schmuck and R. Haskin.: GPFS: A shared-disk file-system for large computing clusters. In Proceedings of the 2002 Conference on File and Storage Technologies (FAST), USENIX, Jan. 2002. 